\title{
A Facility for Testing High-Power Electric Propulsion Systems in Space - A Design Study
}

\author{
Andrew J. Petro \\ Advanced Space Propulsion Laboratory \\ JSC/NASA \\ 13000 Space Center Blvd. Houston, TX 77059 \\ 281-792-5676 (voice) \\ $281-792-5661$ (fax)
}

\begin{abstract}
This paper will describe the results of the preliminary phase of a NASA design study for a facility to test high-power electric propulsion systems in space. The results of this design study are intended to provide a firm foundation for a subsequent detailed design and development activities leading to the deployment of a valuable space facility supporting the new vision of space exploration.
\end{abstract}

The objectives for human and robotic exploration of space can be accomplished affordably, safely and effectively with high-power electric propulsion systems. But, as thruster power levels rise to the hundreds of kilowatts and up to megawatts, their testing will pose stringent and expensive demands on existing Earth-based vacuum facilities. These considerations and the access to near-Earth space provided by the International Space Station (ISS) have led to a renewed interest in space testing. The ISS could provide an excellent platform for a space-based test facility with the continuous vacuum conditions of the natural space environment and no chamber walls to modify the open boundary conditions of the propulsion system exhaust.

The platform would be designed to accommodate the side-by-side testing of multiple types of electric thrusters currently under development and thus provide a strong basis for comparing their relative performance. The utility of testing on the station is further enhanced by the human presence, enabling close interaction with and modification of the test hardware in a true laboratory environment. These conditions facilitate rapid development and flight certification at potentially lower cost than with conventional Earth-bound facilities. As an added benefit, the propulsive effect of these tests could provide some drag compensation for the station, reducing the re-boost cost for the orbital facility. While it is expected that the ISS will not be capable of generating continuous levels of high power, the utilization of state-of-the-art energy storage media would be sufficient to achieve very high power levels over intervals short enough to be feasible and long enough to provide ample demonstration of steady-state operation.

This paper will outline the results of the preliminary phase of the design study with emphasis on the requirements that will dictate the system design. These requirements will be based on the collaborative work of the Electric Propulsion Branch at the NASA Glenn Research Center, the Propulsion Research Center at the NASA Marshall Space 
Flight Center and the Advanced Space Propulsion Laboratory at the NASA Johnson Space Center along with International Space Station program representatives.

Furthermore, the requirements will reflect the inputs of the broader electric propulsion development community including those at universities, commercial enterprises and other government laboratories. As a facility on the International Space Station, the design requirements are also intended to encompass the needs of potential international users.

High-power electric propulsion systems now in development can effectively meet the challenges of human and robotic space exploration with affordability, reliability and safety and enable missions not possible with other propulsion systems. These systems can operate with extremely low propellant consumption thus dramatically reducing the initial launch mass, vehicle scale and cost. They do not require combustion, have few, if any, moving parts and, in some cases, do not require electrodes. These are features that extend system lifetime and increase reliability. High-power electric propulsion systems will significantly reduce trip times and thereby minimize crew exposure to radiation and micro-gravity conditions. Since electric propulsion systems typically provide continuous thrust, the opportunities for mission aborts and other contingency operations are greatly improved, especially if the propulsion system can adjust its thrust and specific impulse (Isp) as needed.

Testing of high power electric propulsion technologies will pose stringent and expensive demands on existing Earth-based facilities. For power levels in the megawatt range, pumping speeds in excess of $10^{6}$ liters per second will be required in order to maintain acceptable conditions in the exhaust region. As illustrated in Figure 1, a thruster using Xenon propellant, for example, operating at $5000 \mathrm{sec}$ Isp or less, could not be tested above 100 kilowatts. Thrusters using lighter gases are of particular interest for future exploration missions and as shown in Figure 1, their testing is even more severely limited even at modest power levels. In addition, the presence of chamber walls modifies the open boundary conditions of plasma rockets, affecting the measurements and hence the assessment of the true system performance. These considerations and the growing human access to near-Earth space, as compared to two decades ago, have led to a renewed interest in space testing.

High-power electric propulsion systems for deep-space missions will likely be powered with electricity supplied by a nuclear reactor. However an important feature of these propulsion systems is that they can also be tested and operated with solar-generated power when that is appropriate. As a facility for testing electric propulsion devices, the ISS could provide a structural attachment, a supply of electrical power, a data link for control and measurements, thermal management, a means to observe the system performance and access for occasional servicing. If a propulsion device needs to be tested at a power level greater than the Station can provide continuously, energy can be stored for periodic testing. 


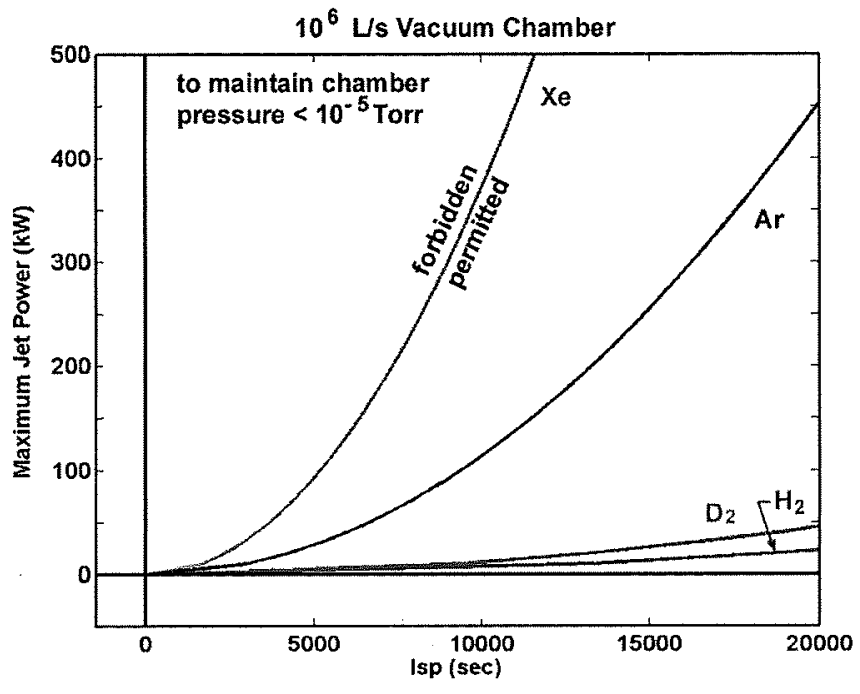

Figure 1: The diagram shows the very limited capabilities of Earth-based vacuum chamber testing for high power electric propulsion systems.

Testing of electric propulsion systems on the space station will help advance the development of systems needed for exploration and could also serve the needs of other customers. Propulsion systems being developed for commercial and military applications could be tested and certification testing of mature thrusters could be accomplished in the space environment. 